\title{
How To Improve MRP Systems To Reduce Cost And Deliver Satisfaction To Customer: A Case Study
}

Mehmet C. Kocakulah (E-mail: mkocakul@usi.edu), University of Southern Indiana Marvin Albin (Email: malbin@usi.edu), University of Southern Indiana Ben Patterson

\begin{abstract}
:
This paper is report of an application integrated in an MRP system and implemented to reduce inventory and its costs. The current system problems were the time needed to develop order packages, abiding by the business rules for order packages, and the animosity the current methods was creating between the supply chain and manufacturing. A package tool was created to help solve these problems. The package tool allows users to select criteria and view exception reports. Implementing this tool resulted in a dramatic increase in meeting customer requested shipping dates and decreased the amount of time spent developing order packages. Use of the tool also resulted in greater compliance with the business rules. Payback occurred in 3.69 months. This experience proves that creative thinking can result in BRP that pays.
\end{abstract}

\section{Introduction}

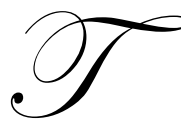

he purpose of this study is to explain an addition that was recently made to Peri Plastics, Structured Product's MRP system. According to Turban [4], MRP, material requirements planning, is software that facilitates the plan for acquiring or producing parts, subassemblies, or materials that allows for the interaction among many products and their components, and the need to change the plan each time that a delivery date or the quantity ordered is changed. McLeod [2] goes on to say MRP software produces several outputs including a planned order schedule, a production schedule, exception reports that flag items requiring management attention, and performance reports. For a good layman's explanation of MRP, see the beginning of Lloyd's [1] MRP (Material Requirements Planning) and MRP2 (Manufacturing Resources Planning) about planning to host a party.

In this explanation, one will find out the benefits that this addition has made for Structured Products in their ability to reduce inventory and its costs, offer On-Time Delivery to customers in order to increase customers' satisfaction, quick response to changing environment, and accurate costing for the processes with their products and services.

\subsection{Peri Plastics}

Peri Plastics is a leader in its industry (Name change for confidentiality purposes). It is made up of many different businesses that all produce different products. One such division is Structured Products. These main businesses are profit centers that contribute to the overall success of Peri Plastics. The various profit centers are given direction to focus on several key corporate initiatives. The two main global initiatives are SPAN and Digitization.

\subsection{SPAN/On-Time Delivery}

SPAN is an internal measurement that focuses on delivering products to customers when they request it. Every order that the customers place with the company has a requested shipping date. One day of SPAN is incurred 
for every day they are late in shipping the products to customers' requested shipping dates. SPAN is measured on every order. If the order ships on time to the customers' requested shipping date, then that particular order will have zero SPAN. SPAN is also incurred for shipping a customer's order prior to the requested shipping date, they placed on the order. The ultimate principle is that if they are able to sustain a low SPAN, then their customers will be happy with the service, thus maintaining their current customer base. Zero (SPAN) is always the company's objective; high SPAN is an indicator of poor customer service and capability. Peri Plastics has reduced its overall business SPAN of from 50 days to less than 5 days in the past year.

In order to better serve their customers, they've focused to maintain a low SPAN to provide more On-Time Deliveries. Usually companies are focusing more on the service side of manufacturing because of the increase in the competition the manufacturing companies are facing. The companies must be able to provide On-Time Delivery as a selling advantage for the customers in order to stay competitive.

\subsection{Digitization}

Peri Plastics also focuses heavily on digitizing current processes. In fact, all employees have been given the charge to automate their jobs. Obviously, all jobs cannot be fully automated, but the expectation is that all employees can digitize certain aspects of the job they hold. During this adventure, many jobs have been eliminated and the focus on other jobs has moved into a more strategic role instead of the day-to-day tactical approach. Digitization is a key to many of the successes that Peri Plastics has realized and continues to benefit from as they move toward a total MRP environment.

\subsection{Structured Products}

While most of the Peri Plastic's subsidiaries make resins, this business buys the resins from other subsidiary and produces extruded sheet and film products; they are a finishing operation. The product line that Structured Products offers is much greater than that of the resin businesses. Therefore, much of their systems are more complicated than the other businesses, thus causing them to be slow in implementing corporate-wide software programs. In fact, many of the corporate systems have to be manipulated once it reaches Structured Products because their business is so much different than the resins businesses.

Structured Products does have a MRP system established, but they are working to further enhance this kind of a system, which they have labeled the Order to Extruder project. The strategic vision of the Order to Extruder project is that an order is placed in customer service department through the web, verified for the raw material availability, scheduled to the correct line, and dropped to the appropriate line so the operators have visibility to run the order. The key to the whole process is that they do not want anyone to touch the order through the entire process. This is a major systematic change from where they are today.

\subsection{MRP Systems}

One of the main reasons for digitizing processes in a manufacturing environment is to create a more satisfying MRP system. According to the article, "What is MRP," [5] a major effect an MRP system should have on manufacturing is that it should accurately predict delivery times, enable faster response times to changing conditions in the market, and it should control every stage of production. The current MRP system combines many software programs and is certainly up to par with the industry, but they feel that it can be enhanced to better serve their customers. See Exhibit 1 for the current MRP system.

The focus on accurate delivery times is a piece of MRP system that can be more fully developed to provide more accurate information to customers. The current process is that MRP system acknowledges every order with a shipping date that is feed back to the customers. This date comes from the lead-times they have on the products in the MRP system. Unfortunately, the lead-times in the system are often inaccurate and do not consider the amount of load on the lines, it is just a static number of days, that does not change unless they go into the system and change 
them. The problem is that they never really feel confident in the number of days it will take before they can make the orders.

When they know they will not hit those delivery dates, they have to go into their system and change the date, which is labeled as a confirmed miss to the customer. The customer receives a fax with the new promised date that they will ship the order. If they were able to more accurately produce the orders on time, or if their lead-times were more accurate and changed based on the load of the lines, they would have less confirmed misses to the customer.

\section{What Is An Order Package?}

An Order Package is the combination of orders that are to be scheduled on a given production line for a specified time Period. These orders are selected from the entire backlog of orders, broken down into the primary lines that the orders run on. For example, S3, a line in Structured Products, can only run blocks QAA and QCC. Therefore, the only orders that can go in the Order Package for line S3 are orders that fall into the QAA or QCC block. An order is placed into a block based on certain characteristics, such as the type of product, gauge, width, and length. Similar products are placed within the same block. They only have the capability to run certain products or blocks on only one line, while others can be run on multiple lines. A sample of their block to line relationship tables is shown in Exhibit 2.

\subsection{Current Methods Of Creating The Order Package For The Lines}

After the determination for which line will run the orders, the schedulers then schedule the orders they want to run based on certain business rules. The business rules for selecting the orders to run as defined by corporate leaders in PERI Plastics are as follows:

- $\quad$ Never run any early orders if you have late orders on that line.

- $\quad$ Target the orders so that the customer knows when he/she can expect the product to ship.

- $\quad$ Never Miss the Confirmed date given to the customer.

- $\quad$ Daily reschedule to include new orders, as appropriate.

- $\quad$ The first 24 hours of the schedule is frozen.

- $\quad$ Never aggregate orders of similar kind if you have late orders assigned to the same line.

- $\quad$ Never be late on producing a Dashboard Order.

- $\quad$ Never be late on producing a Stock Order.

This applies to the scheduling of the orders as well as the execution of making the orders. The ultimate intent in providing these rules is that they want to increase customer satisfaction through delivering the orders to the customers on time. Management feels that they can increase market share by satisfying their current customers with On-Time Delivery while continuously seeking new customers.

As one can see, scheduling the correct orders based on the business rules is not an easy task. There are many orders, and the current method of scheduling to the business rules can be somewhat confusing. It is a very manual task to make sure the correct orders are scheduled, and it is also a time consuming task to ensure that the business rules are followed. Exhibit 3 shows the amount of time involved in determining the correct orders to be scheduled for all of the Structured Products flow streams in the current method of creating order package. A flow stream is defined as a combination of similar products. Film is one of their main flow streams; it is very thin products of extruded plastic that ranges from .001inches to .059 inches in its thickness, while sheet is the other main flow stream that ranges from .060 inches to .5 inches.

Sheet is a very basic form of extruded plastic that their customers use in applications such as signs for advertisements; it receives only a slight layer of coating that is placed on the product at the time it runs on the line. Some of their customers demand a stronger, more durable coating that takes place in finishing department. Finishing is the operation that utilizes some of the products made on the sheet side and applies various thicker 
coatings to offer a more scratch resistant plastic. A common application of a finishing product is the Harley Davidson windshields. The film coating flow stream applies some film products and applies a thicker coating to it. The other flow stream is Multiwall. The common application for Multiwall is the material on the outside of greenhouses. It is a multilayered product that is a good source for heat.

As seen in Exhibit 3, they currently have six resources that allocate portions of their time to deciding the correct orders to be scheduled. Once the decision is made, the Order Packages for the lines are then given to schedulers to schedule to the lines. Then, manufacturing sequences the orders that are scheduled in the most efficient form. Manufacturing looks at the orders given to them, and will sequence them based on the customers' requested shipping dates, and they also balance the amount of changeovers that have to happen to complete the products. A change over is the shifting of the line to another setup to make a different product. The significance of the changeovers is that they are extremely time consuming and are known to be "yield busters". Therefore, manufacturing wants to keep the number of changeovers on the line to a minimum.

\subsection{Problems Encountered In The Current Methods}

One of the main issues encountered in the current method of creating Order Packages is the amount of time it takes to develop the Order Packages. All of the people in Exhibit 3 were assigned to develop these Order Packages because of the recent development of the above mentioned business rules. In other words, the time they are spending on developing these packages for the lines is time taken away from what they were originally hired to do and are measured on.

The scheduler will not schedule an order to the extrusion lines unless it is determined that they have the raw materials on hand to produce the order. In the current process, the people creating the Order Packages had to check the orders for the raw materials before they looked at it to determine if it fit the business rules. The time spent on verifying the raw material availability is time that they could be focusing on other issues if they had the process in place that would only show them the orders with the raw material available.

Another main issue with the current method has been ability to follow the business rules. When a person is looking at hundreds of orders and determining which should be scheduled based on the business rules, mistakes can happen. As a matter of fact, they were all making mistakes weekly because they were not able to give it the proper time due to the other issues going on in their current job responsibilities; therefore, they would do just enough to get by when creating the Order Packages. Any time a process has much manual input mistakes are likely to occur.

Some of the people creating the Order Packages were having difficulty following the new business rules. They were not able to fully make the changes in their mindset from the old method of scheduling. They felt that others would be hindering the net plant yield due to the additional changeovers the new process was asking manufacturing to complete. They even made comments such as the business will fall apart if they are not able to maintain current yield. It was obvious who was following the new rules and who was still operating under the old mindset.

Also, the old process of creating the Order Packages was creating animosity between the Supply Chain and manufacturing. The main reason for this is due to the conflict in measurements. The Supply Chain was looking to better serve the customer and drastically reduce SPAN, while manufacturing was measured on the yield for the lines. As mentioned earlier, the amount of changeovers increased due to the changing business rules to better serve the customers. Although the business realized this would decrease net plan yield, the management maintained the same expectation that manufacturing would produce at least $75 \%$ yield, which they were struggling to make before the changes in business rules.

\subsection{The Need For The Order Package Tool}

As one can easily see, the business needed some form of an automated tool that could create the Order Packages. By having an automated tool, the people creating the manual Order Packages were freed up to focus on 
the jobs they were hired to do. Also, an automated tool could strictly follow the business rules without bias. An automated tool would also provide the customers better service due to the additional amount of time the resources would have to focus on the customer in their current jobs.

\subsection{What Is The Order Package Tool?}

The Order Package Tool is a computer-generated software that was developed to digitize the process of making the Order Packages for scheduling. As mentioned earlier, the current method of creating the Order Packages took approximately ten and a half hours to complete daily. The automated version only takes approximately one hour for one of the schedulers to complete for all of the Structured Products flow streams.

The automated version, the Order Package Tool, screens the entire backlog and determines what orders should be scheduled given the business rules that are to be followed. The Order Package Tool contains the entire backlog for each line. It is a very flexible tool that can be manipulated easily to meet the changing business rules. If the company is operating under a "sold out" condition in which the lines have orders that far exceeds the capacity to run the orders, the Order Package Tool will be run using different parameters than if they had available capacity.

The parameters that are entered for each line when running the Order Package Tool are the Minimum SPAN, Maximum SPAN, Minimum Block Size, Package Duration, and Changeover Time. The scheduler has the flexibility to Change any of these parameters as needed. An example of the parameters screen in developing an Order Package is shown in Exhibit 4. Usually, they run a five-day package for all lines.

\section{The Order Package Tool Logic}

\subsection{The Order Package}

The Order Package Logic is depicted in Exhibit 5. The highlighted orders are the ones that made it into the Order Package for that particular line. The parameters are listed at the top of the exhibit. In this example, the scheduler ran a three day Order Package for that line with a Minimum SPAN $=-10$ days, a Maximum SPAN $=5$ days, a Minimum Block Size $=48$ hrs, and a Standard Changeover time of 4 hours per block. The logic is as follows:

1. All orders that have the raw materials/resin above the Maximum SPAN parameter automatically go into the Order Package unless the total run time is greater than the package duration. If the Order Package Tool has fulfilled its duration, then the orders that did not make it into the Order Package for that line will be labeled in the Order Package Exception Report, as "Package is Full". If an order were above the Maximum SPAN parameter without the raw materials/resin, the order would show up in the Order Package Exception Report with a message indicating the "order does not have the resin". All other orders above the Maximum SPAN will enter the Order Package.

2. Then, the amount of blocks is added up for the amount of orders that are above the Maximum SPAN that enter the package. The number of blocks is multiplied by 4 hours and added to the total run time for orders that made it into the Order Package.

3. All orders below the Minimum SPAN parameter will not go into the Order Package; they will enter the Order Package Exception Report with a message indicating these orders are "Less than the Minimum SPAN parameter".

4. The orders between the Max SPAN and the Minimum SPAN are in the Aggregation Window. These orders are able to make it into the Order Package only if their block is included in the Above Max SPAN or if they are able to aggregate with orders that are in the same block in the Aggregation Window that total to at least the Minimum Block Size. If the block size is less than the Minimum Block Size and the block does not already appear above the Max SPAN then the order will not make it into the Order Package Tool. Instead, these orders will be found in the Order Package Exception Report labeled as "Does not meet the Minimum Block Size". 
An important note is that the orders are all sorted by the "Latest dates", which means that they are trying to run the orders that are the latest to the requested shipping date the customers gave them. The Order Package Tool starts with the latest orders and works its way down to the Minimum SPAN parameter set by the scheduler. It is trying only to run the "Latest dates" for the business, but they rarely ever do that due to a lack of raw materials and the minimum block size that is set.

The QQQ, RRR, and YYY orders did not make it into the Order Package for that line because they did not meet the Minimum Block size. The AAA and the two CCC orders in the aggregation window did make it into the Order Package because their block was already in the Order Package because there were orders in the AAA and CCC blocks above the Maximum SPAN parameters. In other words, since manufacturing was already forced into these set-ups, they would rather run other orders in this set-up, than to make manufacturing run another block. Therefore, increasing output and not negatively affecting yield as bad as it could if they made them run another block. The reason they can do this aggregation is because the window in which the aggregation takes place covers orders that are either not late at all or just a few days late.

The overlying logic of the Order Package Tool is that it will give manufacturing process as much time as it can for them to run orders that fall in the same set up as others. But, they will only wait for so long for manufacturing to find orders that are in the same setup, after they reach the threshold of time; they eventually make them get into that setup to fulfill the customer's request. As one can easily see, manufacturing would like to stay in the same setup as long as possible in order to produce the most amount of pounds and to achieve the highest yields. The Order Package Tool will not allow that, instead it makes them jump around in order to service the customers' requested shipping dates.

In the example one may see that a standard Changeover time has been added in to the total runtime for the orders in the Order Package Tool. The logic is set as a standard Changeover of four hours per block. Therefore, in the example in Exhibit 5, there were four different blocks to be run on that line in the next three days. The four different blocks subtracts 16 hours from the package duration. To apply this example to the real world, they are causing manufacturing a loss of 16 hours worth of production in the next three days in order to follow the business rules. In other words they are scheduling manufacturing to be down a total of $22 \%$ of the time in the next three days due to the loss in production time because of the impact in Changeovers.

There are two main pieces of the Order Package Tool. The first is the section labeled the Order Package. An example of an Order Package, see Exhibit 6. It contains the subset of the backlog for a particular line that they are to schedule within a specified time Period. These are the orders that the system has determined they have the raw materials to produce. These orders are the ones the software provides for the schedulers to schedule based on the certain criteria. The first criteria is that, have the raw materials for the orders, the next criteria is that order meets the business rules for them to schedule, and the last main criteria is that the order can and should be made within the timeframe of the Order Package Tool. If the order did not meet any of the above-mentioned criteria, then it is placed in Order Package Exception Report, the next main piece of the Order Package Tool.

\subsection{The Order Package Tool Exception Report}

The Order Package Exception Report shows the remaining orders that the Order Package Tool was unable to fit in the Order Package. All of the orders that are in the Order Package Exception Report are accompanied with an exception message. The exception message concisely explains the reason the orders did not make it into the Order Package. The exception messages are: No resin, Package is full, Order SPAN is less than the Minimum Span parameter, Lot status = void, Lot status = Incomplete, Does not meet the Minimum Block Size, or No Run Rate. This information explains to the scheduler the reason the order did not make it into the Order Package. An example of the Order Package Tool Exception Report, see Exhibit 6.

The scheduler is trained to understand the exception messages. The scheduler knows what to do when she/he sees an exception message that is labeled as "No Run Rate". Since the Order Package Tool is time dependant, if the order does not have a Run Rate in the current MRP system, it can not make it into the Order 
Package because they would be unsure how long it would take to run that order. Therefore, the scheduler knows that she/he is supposed to work with manufacturing to determine a Run Rate for the order and she/he then keys in the Run Rate in the Run Rate Table in the Order Package Tool. After completing this, the scheduler has to rerun the Order Package for that particular line to see if the order that did not have a Run Rate made it into the Order Package or the Order Package Exception Report.

The Order Package Tool will not let orders that do not have the raw materials to make the order enter the Order Packages for the lines. Obviously, the most important raw material for Structured Products is the resin. There are many different kinds and colors of resin. The Order Package Tool is linked to resin inventories to determine if the individual orders have the resin needed to run the order. If the resin is not on hand for a particular order, the Order Package Tool will not let that order enter the Order Package for the primary line associated with that order.

For example, if an order is placed for some 9030 grade material that has a thickness, or gauge, of .030 inches and has a color of 112, which is natural, that order is determined to be in the QAA block by our MRP system. The MRP system looks at the order and determines all of the necessary ingredients that go into making that product through the Bill of Materials. It determines that the resin is 9030T-112, which kept an average inventory level of $1,000,000$ pounds of inventory. Even though, they keep a safety stock of that resin on hand, they frequently run out of stock, due to the sheer volume of orders that require that resin. In this example, if the resin is not on hand, the order will go into the Order Package Tool's Exception Report for Line S3 and the exception message would read "no resin in inventory".

An order in the Order Package Exception Report that receives the exception message of "Order SPAN is less than the Minimum SPAN" is an order that is too far out in the future. This basically means that, business does not want to look at orders that are more than two weeks before the requested shipping date. The reason for this is that if they continually look that far out in advance, it will be aggregating some of the early orders before running all of their late orders for manufacturing efficiencies, which directly violates one of the corporate mandated rules.

Another exception message reads, "The Order Package is Full". This is the message that accompanies the orders that the Order Package Tool wanted to bring in and run, but were unable to do so based on the amount of orders already in the Order Package and the amount of time left to fulfill the package duration. The package duration parameter is set with a tolerance of plus or minus ten percent. As one may have noticed in Exhibit 5, the package duration parameter was set at 72 hours, and the order Package totaled 73 hours. It was able to do so because of the plus and minus ten percent set range.

One of the drawbacks to the Order Package Tool is that it only has the knowledge to look at what primary line is associated to the block. If one line does not have a full package and another line has orders in the Order Package Exception Report that have an exception message of "Package is Full", then the scheduler can move some of the orders around to another line in order to run all of the orders that the Order Package Tool would have liked to run within a certain time Period. Scheduler has the knowledge of where the products run because of the block to line relationship that was referred in Exhibit 2. This is manual work that the scheduler has to do because the logic associated with building a tool that could handle the load and the mix of the load was too complex and costly for the business to look at other lines for the blocks that can run on several lines.

The reason the orders that have a Lot Status of Incomplete and Void are placed in the Order Package Tool Exception Report. Because something is wrong with these orders that caused them to either void the scheduled Lot or call it Incomplete. They did not want to include these in the Order Package for the lines due to the numerous reasons orders could have this status. These have to be dealt with on a case-by-case basis. Therefore, the scheduler looks at these in various systems to find out the root cause for the orders to appear with either of these statuses. During the research process, she/he may choose to reschedule them, cancel the order, order more resin, or do many other things. 
The last main exception message that shows up in the Order Package Exception Report is "does not meet the minimum block size". As mentioned earlier, this message accompanies any order in the aggregation window that was not in the blocks in the orders above the Maximum SPAN or did not have enough similar orders for that block in the aggregation window. The main reason they would not want to run these, because they are trying to give manufacturing time to get in like orders in the books for the same block so that it can minimize the amount of times they have to go in and run that block for manufacturing efficiencies.

\section{Benefits Of The Order Package Tool}

\subsection{Decrease In SPAN}

SPAN is a unique measurement. The way SPAN is calculated is determining the SPAN on every order that ships to customer. Then, they knock off the top two percent of highest and lowest SPAN items. Therefore, they are only counting SPAN on $96 \%$ of the orders that shipped within a fiscal week. The highest SPAN item left in the 96\% is the number that is reported out as the overall business's SPAN. It is more complicated by adding in the early shipments to the latest shipments to make the overall SPAN number for the business. For example using the 96\% data, if they shipped latest order, which totaled 20 days late in the $96 \%$ of the data, and they also shipped an order 2 days early in the same dataset, then their overall business SPAN would be 22 days. All PERI Plastics' businesses report out their SPAN numbers that are placed on the PERI Plastics' home page, so the corporate management can keep their eye on their businesses.

On April 11, 2001, Structured Products was diagnosed as the highest SPAN business in all of PERI Plastics. Their SPAN numbers were around 17 days. They were receiving much pressure to bring SPAN numbers down drastically and quickly. So they focused on every process that they could to reduce SPAN. They have been working on the development of this tool for the past year. They received much pressure to implement this tool as quickly as possible.

They implemented this tool in the beginning of May. As soon as this tool was released, they noticed a drastic improvement in their SPAN performance. See Exhibit 7 for the results in the achievements of the Order Package Tool along with other small projects. Part of the reason they were able to see such results was the fact that resources were reallocated back to certain aspects of their jobs to focus on improvements in processes to reduce SPAN because the need for their work on creating the Order Packages was eliminated.

\subsection{Headcount Reduction}

As mentioned earlier, they were able to take the existing process and develop a digitized version of doing the job. Thus, they were able to reduce about nine hours worth of work per day involved in creating Order Packages for the lines. Because of this reduction, they were able to reduce from two schedulers to one because of the Order Package Tool. They offered an early retirement to one of the schedulers. The cost savings of this is estimated to be minimum $\$ 65,000$ a year.

\subsection{Other benefits}

They will be able to clearly see the effect a hot sheet, or a short requested lead-time, given to them by their customer has on the other orders they have scheduled. In the past, company accepted hot sheets and short requested lead-times without looking at the implications it would cause the other orders. By adding in a hot sheet to a line, company may miss six customer orders that have had their orders on the books up to months in advance. This was not a desired process, and through the Order Package Tool, the company can easily see the effect a hot sheet has on the rest of the Order Package. They also be able to manually insert an order into an Order Package by typing in the order number and clicking on a must include flag. See Exhibit 6 for an illustration of how a hot sheet enters an Order Package. Clicking on the "Include" button and typing in the customer order number next to it accept a hot sheet. All the scheduler has to do in order to see the effect that an order has on the overall Order Package for that line is to run the Order Package Tool before including the hot sheet and check that to the new Order Package that is 
run with including the hot sheet. The difference in the Order Package is the effect the hot sheet will have on that Order Package.

The company also attributing the Order Package Tool with business knowledge those only key individuals had on the processes of creating the Order Packages. If several of these key people left the business, they would have been in serious trouble because much of what they did was in their heads without being written down. Companies can be in serious trouble when people who hold key knowledge leave the company. With the Order Package Tool, anyone can run the packages, and once they use it and run packages for a couple of days, people become familiar with the logic and the application. Having this flexibility in the today's shaky economic times certainly a plus.

The Order Package Tool is also been beneficial for manufacturing to hold the operators accountable for the amount of time it takes them to run orders. The Order Package Tool assigns all orders with Run Rates. Because of this, the company able to have a measuring sticks for manufacturing to complete the orders within a given time frame. If it takes them longer to run an order than what the Order Package Tool calculated, then questions are asked and people are looking into why it took longer than it is supposed to take to make the product. Having a measuring devise has helped not only company's yield, but also the amount of pounds that they are capable of producing. Certain resources have been deployed to tackle the slow manufacturing products. Alfred Peterson [3], the Chairman of Peterson American Corporation, states, "unfortunately, the startling progress some companies are making in their operations through the use of such new types of measurements only represent pockets of progress in the overall industry". He goes on to state that companies should streamline their Supply Chains in order to provide better products at more attractive prices for the customers [3].

The Order Package Tool has also ensured that following the business rules when creating the Order Packages for the lines. By following the business rules, SPAN has decreased, and the number of Changeovers has increased. Because of the increase in the number of Changeovers, the company has also reallocated resources to focus on decreasing the amount of time it takes to perform the Changeovers, which would reduce the amount of scrap time they've experience in the past.

The Order Package Tool has pointed out that the company has serious problems with their lead-times in the MRP system. Since they know exactly how long it takes the orders to run on the lines from the Order Package Tool, they can become more accurate in giving our lead-times to our customers, which will further enhance our current MRP system. Peri plastics have developed a team to focus on developing an algorithm that will enable the leadtimes to move with the variance in the load of the lines. Currently, they are trying to determine what is the best case scenario for developing all of the products; granted, different flow-streams have different best case scenario dates associated with them due to the variances in the amount of time it takes to produce one product over another.

\subsection{The Order Package Tool's Fit Within The Strategic Vision}

The Order Package Tool is a great enhancement to Peri plastics current MRP systems. It adds extra value that was not already there. To view how the Order Package Tool fits in with their current MRP system, see Exhibit 8. It fits in nicely to the overall business strategy of Order to Extruder, in which a person never touches the order. Order to Extruder is a process in which the customer places the order over the web, and the order flows through the various systems to determine the correct raw materials and packaging for the order. It is then assigned a block, associated with a certain line, passes into the Order Package for that line, becomes scheduled and assigned a Lot Number, falls down to the line it is supposed to run on, falls in the sequences where appropriate, and is run by the operators.

Also during the process of becoming sequenced, the customer is sent a time the order will be completed; therefore, they can plan on the necessary things they need to prepare for when it arrives. Peri Plastics, as a business, are much closer to this process because of the Order Package Tool. They are still at least a year off from the completion of the Order to Extruder project, but they could not finish it in one year if they were without the Order 
Package Tool. The Order Package Tool is a major step in the right direction, and it also proves that extremely complex processes can be digitized.

\section{Summary}

The Order Package Tool development and implementation cost Structured Products $\$ 20,000$, and the dollar savings in this year alone totals $\$ 37,912$. The payback Period is 3.69 months. Not only has this been a good financial project for Structured Products, but is has also proved to be beneficial in many other realms as mentioned above.

The Order Package Tool, has proven to be extremely successful in their business for reducing SPAN, decreasing the time put into creating Order Packages, reducing costs through the reduction in a headcount, forcing them to follow the business rules, and leading company in the right direction in the Order to Extruder project. It has proven that difficult, complex tasks that normally would not consider a good source for digitization can be automated. Also, it provides outstanding data to point out problematic areas or product lines that need resources focused on to improve on the processes. Peri Plastics customers are certainly feeling the effects of the reduction in SPAN through on-time delivery and they have mentioned their satisfaction has grown with Structured Products.

\section{Conclusion}

Many times people get caught up in doing their jobs and do not focus on ways to improve the current methods of doing their jobs. Usually, it is because it would require more time for them to do that, and time is an extremely valuable as well as limited resource. We urge anyone not to follow that mindset because getting stuck doing the same practices that have been done for years without challenging themselves can be a serious detriment to personal satisfaction and the company's productivity. Challenge the practices and methods of doing things, and we believe one will come up with several projects to improve upon the existing practices. This is especially true if one thinks creatively about how IT can be applied if one digitizes a task. By improving the existing practices, one will be able to free up time and money for one's company, thus proving to be a valuable resource for the company and increase job satisfaction for themselves.

\section{References}

1. Lloyd, A., Project Director, Omni Business Facility, The University of Edinburgh Management School. http://omni.bus.ed.ac.uk/opsman/oakland/oak22.htm. June 2000.

2. McLeod, R., Jr. Management Information Systems. 7th ed., pp. 489-90. Upper Saddle River, NJ: Prentice Hall, 1998.

3. Peterson, A. "Supply Chain Growth is Limited by Lack of New Supply Chain Efficiency Measures, and New Legal and Accounting Arrangement", OSAT's Focus on the Future. Spring 1997. http://www.osat.umich.edu/newsletters/news20.html.

4. Turban, E., McLean, E. \& Wetherbe, J. Information Technology for Management. 2d ed., p. 342. New York, NY: John Wiley \& Sons, 2001.

5. "What is MRP?", June 24, 2001. Caliach Limited, Middlesex, England. http://www.caliach.com/ caliach/about/whatMRP.html. 
Exhibit 1: Current MRP System

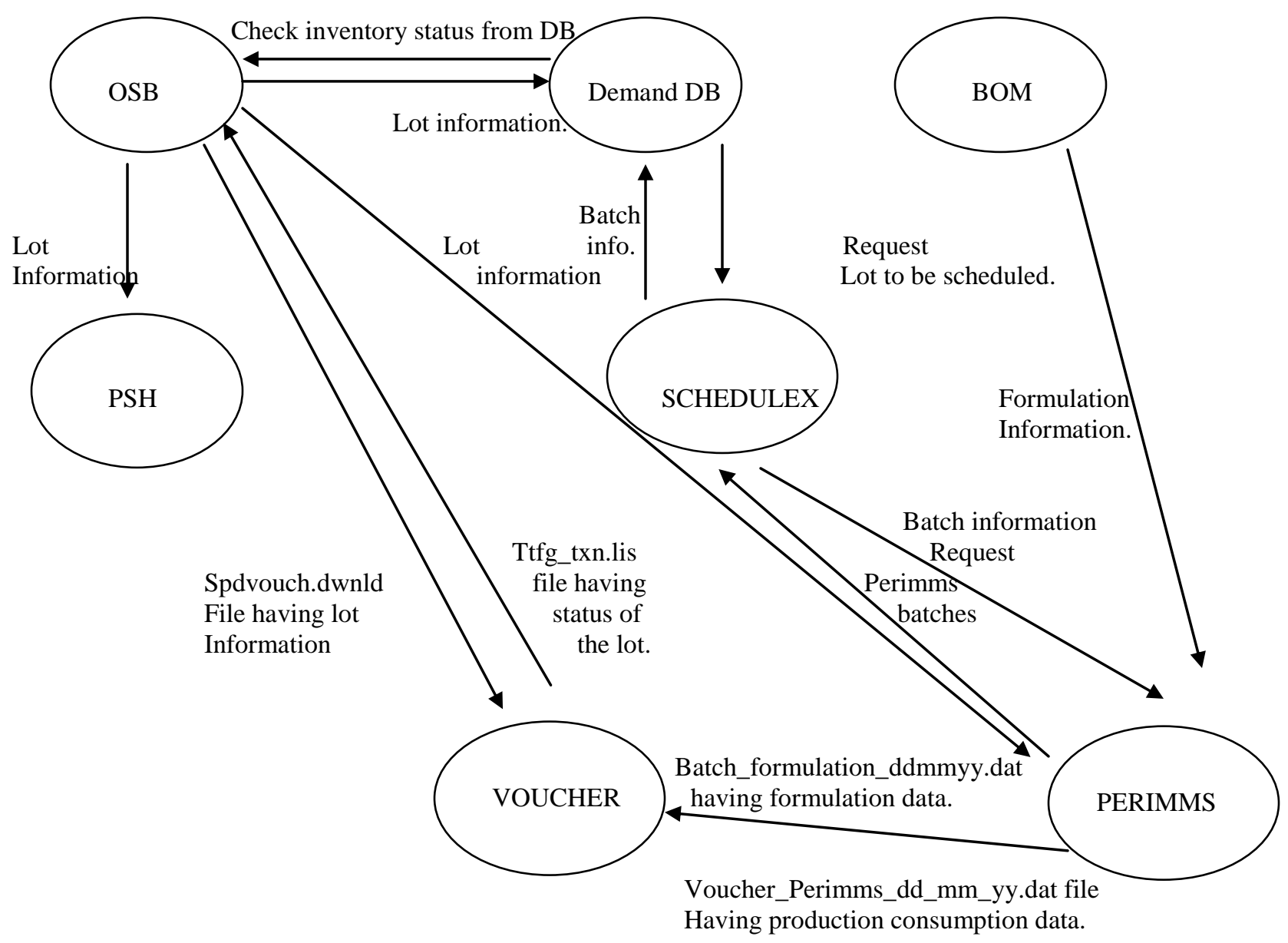


Exhibit 2: A Sample Of Block To Line Table

\begin{tabular}{|c|c|c|c|c|}
\hline Block & Line & Type & Operation & \\
\hline BAA & S5 & 1 & Sheet & \\
\hline BAA & S8 & 2 & Sheet & \\
\hline BAB & S8 & 1 & Sheet & \\
\hline $\mathrm{BAB}$ & S5 & 2 & Sheet & \\
\hline BBB & S8 & 1 & Sheet & \\
\hline BGG & S5 & 1 & Sheet & \\
\hline BGG & S8 & 2 & Sheet & \\
\hline MBA & $\mathrm{C} 41$ & 1 & Mar & \\
\hline MBB & $\mathrm{C} 41$ & 1 & Mar & \\
\hline $\mathrm{MBC}$ & $\mathrm{C} 41$ & 1 & Mar & \\
\hline MBD & $\mathrm{C} 41$ & 1 & Mar & \\
\hline $\mathrm{MBH}$ & $\mathrm{C} 41$ & 1 & Mar & \\
\hline LCB & $\mathrm{C} 42$ & 1 & Fmr & \\
\hline LDD & $\mathrm{C} 42$ & 1 & Fmr & \\
\hline LEE & $\mathrm{C} 42$ & 1 & Fmr & \\
\hline MLA & S14 & 1 & Corr & \\
\hline MLL & P4 & 1 & Corr & \\
\hline MLP & P4 & 1 & Corr & \\
\hline MLQ & P4 & 1 & Corr & As one can easily see, some blocks \\
\hline PAA & S16 & 1 & Multiwall & can run on multiple lines, such as in Film, \\
\hline PEE & S16 & 1 & Multiwall & and others can only run on one line \\
\hline PFF & S16 & 1 & Multiwall & \\
\hline PGG & S16 & 1 & Multiwall & \\
\hline RBB & F6 & 1 & Film & \\
\hline RBB & $\mathrm{F} 1$ & 2 & Film & \\
\hline RBB & F10 & 3 & Film & \\
\hline RBB & $\mathrm{F} 2$ & 4 & Film & \\
\hline RBB & F12 & 5 & Film & \\
\hline RBB & F9 & 6 & Film & \\
\hline RBB & F11 & 7 & Film & \\
\hline $\mathrm{RCC}$ & F6 & 1 & Film & \\
\hline $\mathrm{RCC}$ & $\mathrm{F} 1$ & 2 & Film & \\
\hline $\mathrm{RCC}$ & F10 & 3 & Film & \\
\hline $\mathrm{RCC}$ & $\mathrm{F} 2$ & 4 & Film & \\
\hline $\mathrm{RCC}$ & $\mathrm{F} 12$ & 5 & Film & \\
\hline $\mathrm{RCC}$ & F9 & 6 & Film & \\
\hline $\mathrm{RCC}$ & F11 & 7 & Film & \\
\hline TAA & $\mathrm{C} 45$ & 1 & Coated Film & \\
\hline TAH & $\mathrm{C} 45$ & 1 & Coated Film & \\
\hline TAW & $\mathrm{C} 45$ & 1 & Coated Film & \\
\hline TLL & $\mathrm{C} 45$ & 1 & Coated Film & \\
\hline
\end{tabular}




\section{Expected Benefits}

1) Span: "Control" Mechanism to drive Span down or to at least maintain Span

2) Resources:

\begin{tabular}{|l|c|c|}
\hline & $\begin{array}{c}\text { Currently } \\
\text { \# of hours per day }\end{array}$ & $\begin{array}{c}\text { With Order Package Tool } \\
\text { \# of hours per day }\end{array}$ \\
\hline Pat & 3 & 0 \\
\hline Charlie & 2 & 1 \\
\hline Ben & 3 & 0 \\
\hline Steve & 2 & 0 \\
\hline Bill & 0.25 & 0 \\
\hline Doug & 0 & 0 \\
\hline Total & $\mathbf{1 0 . 2 5}$ & $\mathbf{1}$ \\
\hline
\end{tabular}

-Doug York retired on May 1st.

-Digitization to Schedule $\mathrm{X}$ is included in the above figures 
Exhibit 4: Parameters Screen

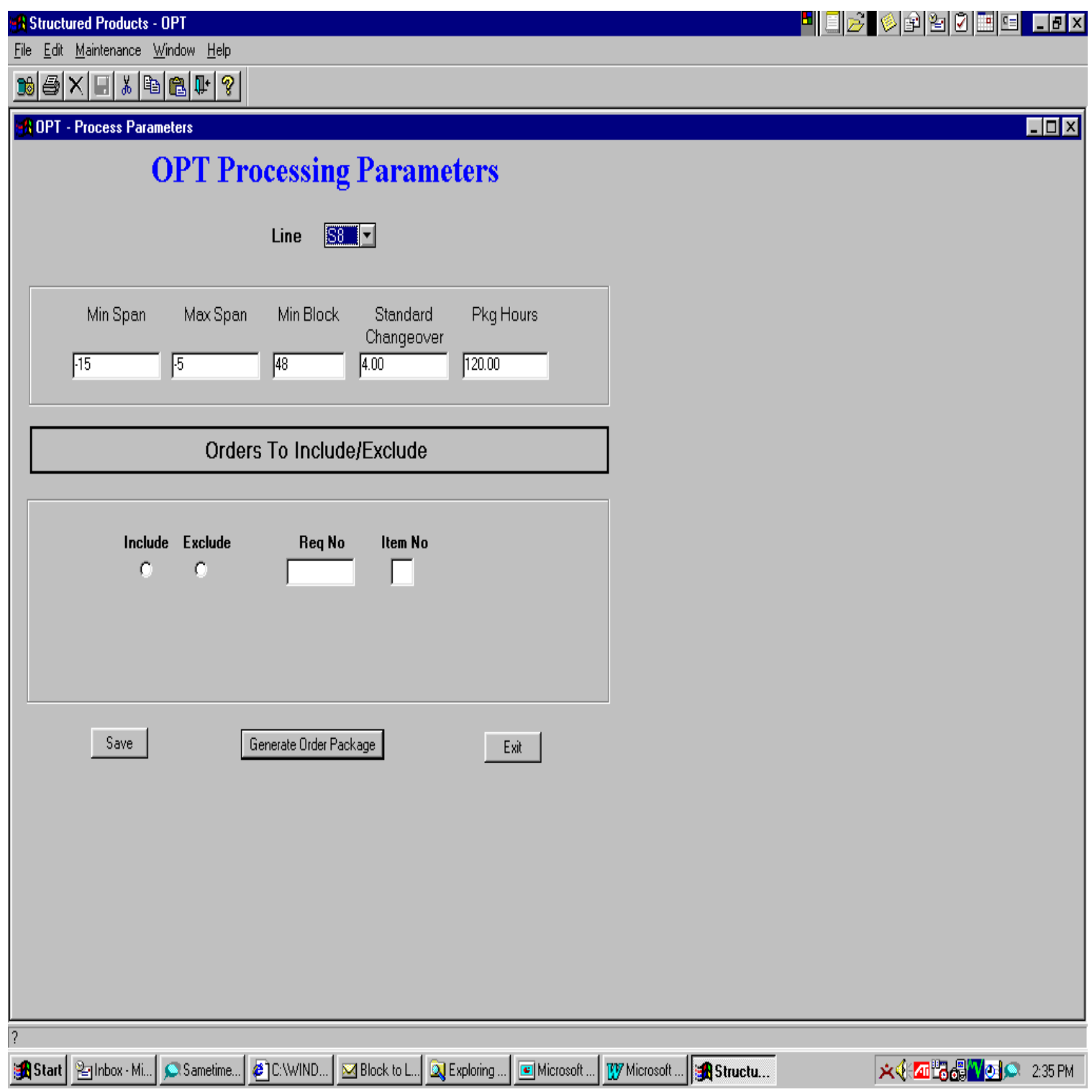


g

\section{Order Package Tool}

QPID 21468

\begin{tabular}{|c|c|c|c|c|c|c|}
\hline & & OP & T Logic $E$ & & & \\
\hline Package Duratic & $n=72 \mathrm{~h}$ & & & & & \\
\hline Minimum Block & Size $=4 \varepsilon$ & & & & & \\
\hline Max Span = 5 & & & & & & \\
\hline Min Span $=-10$ & & & & & & \\
\hline & Block & Run time in Hrs & Span days & & & \\
\hline 4 & AAA & 2.5 & 10 & & & \\
\hline & BBB & 5 & 9 & & Total run time & 40.5 \\
\hline MUST & $\mathrm{CCC}$ & 0.75 & 8 & & 4 blocks $x 4$ hrs per block & 16 \\
\hline MANUFACTURE & DDD & 4.25 & 7 & & Total package & 56.5 \\
\hline & AAA & 16 & 6 & & & \\
\hline 1 & AAA & 12 & 6 & & Span $=5$ days & \\
\hline 4 & $\overline{Q Q Q}$ & 15 & 5 & & & \\
\hline & RRR & 22 & 4 & & & \\
\hline & AAA & 2.5 & 4 & & Total run time & 16 \\
\hline Aggregation & YYY & 12 & 2 & & Change over time & $\underline{0}$ \\
\hline Window & CCC & 7.5 & 2 & & Total package & $\overline{16}$ \\
\hline & YYY & 0.25 & 2 & & & \\
\hline & $\mathrm{QQQ}$ & 5 & 0 & & & \\
\hline & $Q Q Q$ & 5 & -9 & & & \\
\hline & CCC & 6 & -9 & & & \\
\hline$\checkmark$ & RRR & 7.8 & -9 & & Span $=-10$ days & \\
\hline & YYY & 1.4 & -15 & & & \\
\hline & AAA & 48 & -18 & & Total package run time & 57 \\
\hline & AAA & 24 & -19 & & Total change over time & 16 \\
\hline & AAA & 5 & -19 & & Total package & $\overline{73}$ \\
\hline
\end{tabular}


Exhibit 6: Order Package Tool Example For Line S8

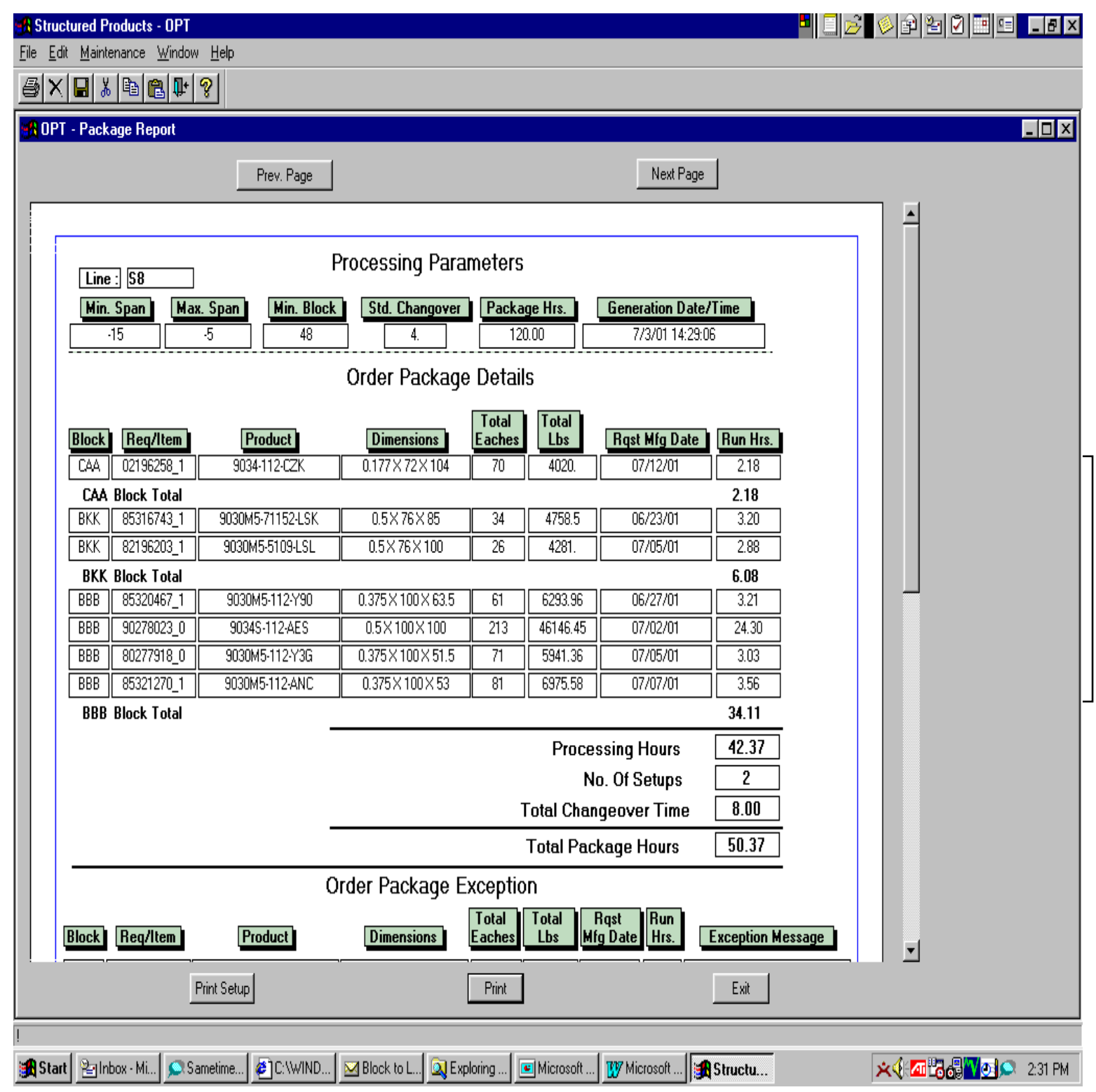


Exhibit 7: Historical Structured Products SPAN Information For 2001

\section{Structured Products Americas}

$\longleftarrow$ P98
$\longleftarrow$ P2
$\rightarrow$ P95 Span
$\rightarrow$ Rolling 4 Weeks span P98 Span

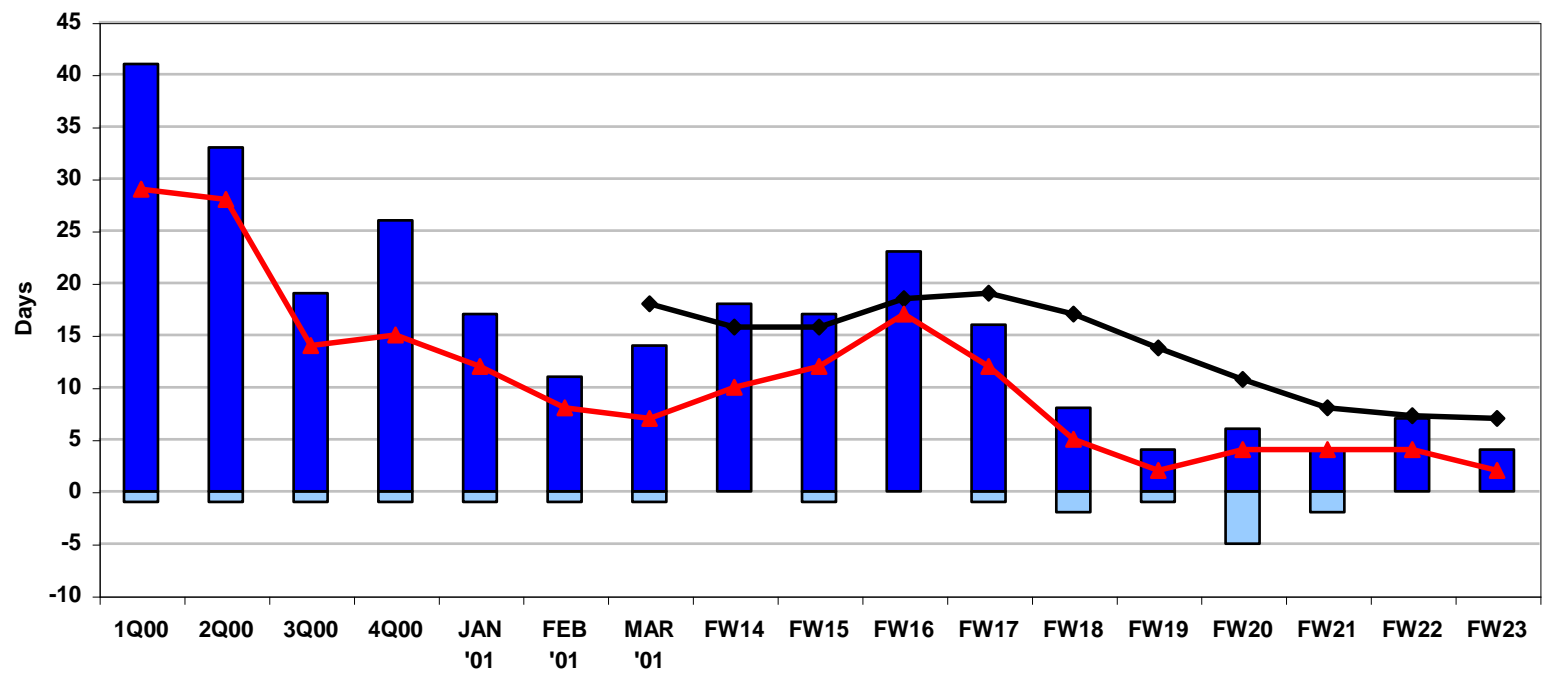


Exhibit 8: New MRP System Changes With The Order Package Tool

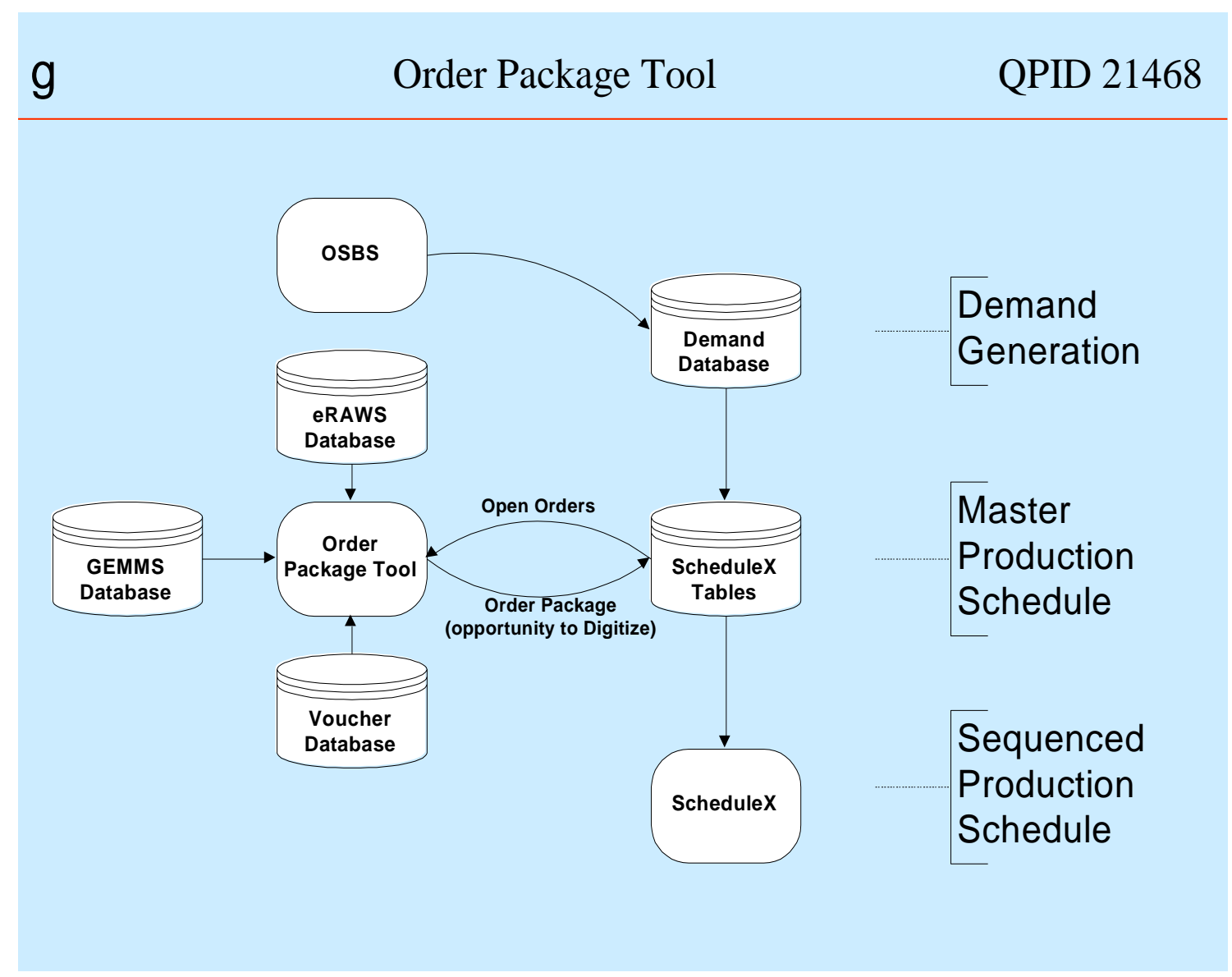

The Order Package Tool integrates systems that are on different platforms. The impact is that they are now able to pass information through the various systems in a more efficient manner, and the Order Package Tool acts as a bridge to intertwine the two different computer platforms with each other so that the information can pass from one platform to another. 